\section{Diffusion of Gold in Solid Lithium}

\section{А. Отт}

Physics Department, Chalmers University of Technology, Gothenburg, Sweden

(Z. Naturforsch. 23 a, 1683 1684 [1968] ; received 5 August 1968)

The diffusion of gold in lithium metal of normal isotopic composition has been measured between $45^{\circ} \mathrm{C}$ and $150{ }^{\circ} \mathrm{C}$, using a thin film plating and sectioning method. The data fit the Arrhenius relation $D=D_{0} \exp (-Q / R T)$ where

$D_{0}=0.21 \pm 0.08 \mathrm{~cm}^{2} \mathrm{sec}^{-1}$ and $Q=10.99 \pm 0.18 \mathrm{kcal} \cdot \mathrm{mol}^{-1}$.

These results are in contrast to those obtained for other impurities in lithium, and can not be readily interpreted by any existing theory.

Measurements of gold diffusion in lithium of the "reversed" isotopic composition show diffusion rates approximately $8 \%$ higher than in "normal" lithium.

In order to shed light upon the phenomena of diffusion in the alkali metals and the dependence of the diffusion parameters on valency, atomic size and electron core differences between solute and solvent atoms, we have undertaken a study of diffusion of gold in lithium metal of normal isotopic composition $\left(92.5 \%{ }^{7} \mathrm{Li}\right.$, $\left.7.5 \%{ }^{6} \mathrm{Li}\right)$ and in lithium metal of the nearly reversed isotopic composition $\left(4.4 \%{ }^{7} \mathrm{Li}, 95.6 \%{ }^{6} \mathrm{Li}\right)$.

This investigations was a part of a systematic study of impurity diffusion in the lithium lattice. We have earlier studied the diffusion of sodium ${ }^{1}$, silver $^{2}$, zink ${ }^{3}$ in lithium and the self-diffusion of lithium ${ }^{4}$. The experimental procedure and evaluation of the present results was similar as in earlier work, and need not be discussed here. The "normal" lithium metal was from the Foote Lithium Corporation. The "reversed" lithium metal was from Oak Ridge. Both metals were of 3N5 grade. The ${ }^{195} \mathrm{Au}$ was from the New England Nuclear Corporation and free from radioactive impurities.

The experimental values of the diffusion coefficient, $D$, are given in Table 1 and plotted in Fig. 1 .

\begin{tabular}{rrcr}
\hline$T\left({ }^{\circ} \mathrm{C}\right)$ & $D \cdot 10^{9}\left(\mathrm{~cm}^{2} / \mathrm{sec}\right)$ & $D^{\prime} \cdot 10^{9}\left(\mathrm{~cm}^{2} / \mathrm{sec}\right)$ & $D^{\prime} / D$ \\
\hline 152,5 & $460.6 \pm 10.5$ & & \\
144.0 & $357.5 \pm 7.5$ & & \\
137.0 & $261.9 \pm 3.0$ & & \\
125.9 & $200.2 \pm 4.0$ & $215.2 \pm 4.0$ & 1.07 \\
113.8 & $118.5 \pm 2.5$ & & \\
106.7 & $92.87 \pm 2.0$ & & \\
98.1 & $60.87 \pm 1.5$ & $65.69 \pm 1.5$ & 1.08 \\
85.2 & $38.81 \pm 1.0$ & $42.90 \pm 1.3$ & 1.10 \\
78.9 & & $30.05 \pm 1.5$ & \\
75.8 & $27.05 \pm 0.05$ & & \\
54.5 & $10.18 \pm 0.15$ & & \\
49.8 & $7.579 \pm 0.02$ & & \\
46.8 & $5.801 \pm 0.15$ & & \\
\hline
\end{tabular}

Table 1. Experimental Results. $D$ denotes the diffusion coefficient in normal lithium, $D^{\prime}$ that in lithium with $95 \%{ }^{6} \mathrm{Li}$.

1 J. N. Mundy, A. Ott, and L. Löwenberg, Z. Naturforsch. 22 a, 2113 [1967].

2 A. Ott and A. NoRdÉn-Ott, Z. Naturforsch. 23 a, 473 [1968].

3 J. N. Mundy, A. Otт, L. Löwenberg, and A. Lodding, to be published.

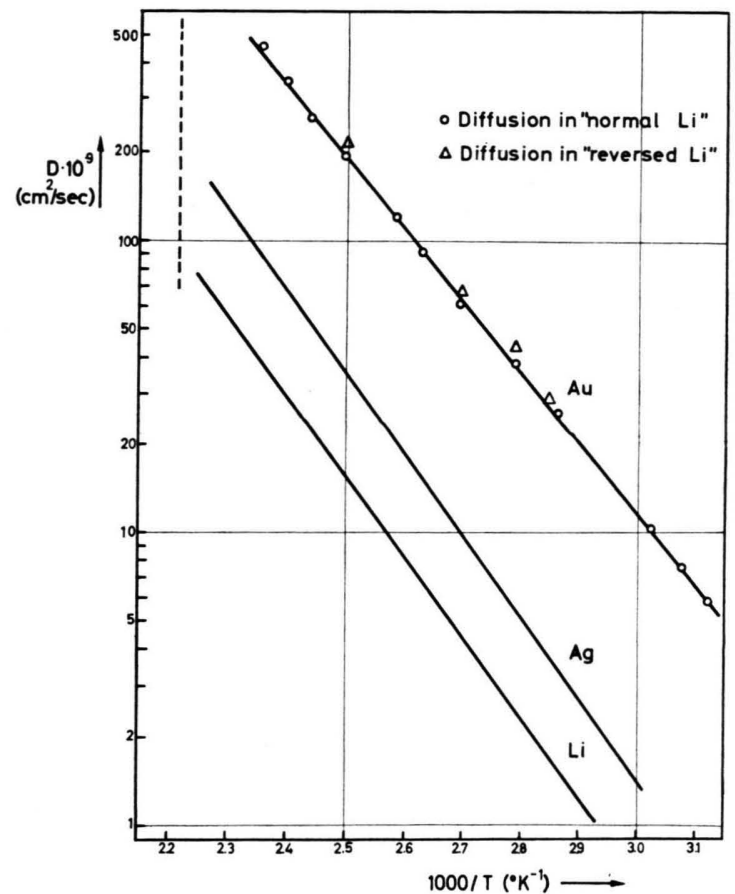

Fig. 1. Arrhenius plot of diffusion in lithium metal. The present work is here compared with the diffusion results for $\mathrm{Ag}^{110 \mathrm{~m}}$ and ${ }^{6} \mathrm{Li}$ tracers in $\mathrm{Li}$.

From the slope in the $\log D$ versus $1 / T$ diagram we obtained the activation energy $Q$, and from the intercept on the $1 / T$ axis we got the $D_{0}$ term in the Arrhenius equation for $D$. These are listed in Table 2 together with the other known values of diffusion parameters in lithium.

\begin{tabular}{lccc}
\hline & $D_{0}\left(\mathrm{~cm}^{2} \mathrm{sec}^{-1}\right)$ & $Q\left(\mathrm{kcal} \cdot \mathrm{mol}^{-1}\right)$ & Reference \\
\hline $\mathrm{Li}$ in $\mathrm{Li}$ & $0.12 \pm 0.05$ & $12.62 \pm 0.21$ & 4 \\
$\mathrm{Na}$ in Li & $0.41 \pm 0.09$ & $12.61 \pm 0.15$ & 1 \\
$\mathrm{Zn} \mathrm{in} \mathrm{Li}$ & $0.57 \pm 0.30$ & $12.98 \pm 0.24$ & 3 \\
$\mathrm{Ag}$ in Li & $0.37 \pm 0.13$ & $12.83 \pm 0.25$ & 2 \\
$\mathrm{Au}$ in Li & $0.21 \pm 0.08$ & $10.99 \pm 0.18$ & \\
\hline
\end{tabular}

Table 2. Impurity diffusion data in lithium.

Supposing that the gold tracer is univalent, the homovalent theory by LECLAIRE ${ }^{5}$ can be applied to the present results. This theory aims, as do other theories of impurity diffusion in solid metals, at calculating the difference $\Delta Q$ between the activation energies of impurity respectively self-diffusion:

$$
\Delta Q=Q_{2}-Q_{0} \text {. }
$$

4 A. Ott, J. N. Mundy, L. Löwenberg, and A. Lodding, Z. Naturforsch. 23 a, 771 [1968].

5 A. D. LeClaire, Phil. Mag. 10, 641 [1964]. 
$Q_{2}$ and $Q_{0}$ are the activation energies for solute resp. solvent atoms.

The treatment entails the calculation of a perturbation potential $U$ at the impurity atom. A positive $U$ means a potential "well" at the impurity atom, electrons are attracted into this well, charging it negatively, and thus repelling vacancies, which in a monovalent metal have an effective negative unit charge. If $U$ is negative, electrons are repelled from the impurity atom, vacancies are attracted and diffusivity enhanced. $U$ is not directly comparable with $\Delta Q$, but they should be of the same sign. This potential $U$ is expressed as a function of the heat of sublimation, the mean Fermi energy and the ionization potentials for the solute and solvent. In our experiment we find that $\Delta Q$ is negative, but the theoretical potential $U$ is positive. This discrepancy for the homovalent theory might indicate that gold dissolved in lithium is ionized to a higher order than the first.

As gold may also occur with the chemical valency of three, it should be interesting to regard the consequence of gold as a heterovalent impurity with a valency difference of +2 and to apply the LeCLAIRE heterovalent theory ${ }^{6}$ to such a case. According to this theory, and assuming a vacancy mechanism for diffusion, the difference in activation energy for the solute and solvent $\Delta Q$ can be expressed as

$$
\Delta Q=\Delta E_{\mathrm{f}}+\Delta E_{\mathrm{m}}-C .
$$

$\Delta E_{\mathrm{f}}, \Delta E_{\mathrm{m}}$ are the solute-solvent differences in formation resp. motion energy of an adjacent vacancy. $C$ accounts for the temperature dependence of the impurity correlation factor $f$.

Performing the arithmetic operations ${ }^{7}$ we get

$$
C=\frac{\Delta E_{\mathrm{m}}}{1+2.27 w_{0} / w_{2}} .
$$

If gold is three-valent in lithium, the coulombic attraction between a gold atom and a vacancy might make the jump frequency $w_{2}$ considerably higher than the jump frequency $w_{0}$. This, however, would make $C$ about the same as $\Delta E_{\mathrm{m}}$ and $\Delta Q$ will be about equal to $\Delta E_{\mathrm{f}}$.

6 A. D. LeClaire, Phil. Mag. 7, 141 [1962].

7 L. W. Barr, J. N. Mundy, and F. A. Smith, Phil. Mag. 16, 1139 [1967].

8 K. Umeda and S. Kobayashi, J. Phys. Soc. Japan 13, 148 [1958].
According to LeClaire $\Delta E_{\mathrm{f}}$ can be evaluated from the expression

$$
\Delta E_{\mathrm{f}}=-2 \alpha Z\left(e^{2} / a\right) \cdot \exp (-q a)
$$

where $(-Z e)$ is the excess charge of the impurity, $e$ is the unit electronic charge, $a$ is the nearest neighbour distance, $\alpha$ is a constant of the order of unity ${ }^{8}$, $q$ is the screening parameter $\left(4 \pi N_{\mathrm{e}}\right)^{1 / 2}$, where $N_{\mathrm{e}}$ is the density of states at the Fermi level. For $\mathrm{Li} q$ is about $1.6 \AA^{-1}, a$ is $3.1 \AA$. Using the valency $Z=+2$ for $A u$ we get $\Delta E_{\mathrm{f}}$ to about $-1.8 \mathrm{kcal} / \mathrm{mole}$. This is in acceptable agreement with the experimental value of $-1.6 \mathrm{kcal} / \mathrm{mole}$.

There are reasons to believe, however, that any quantitative agreement with LeClaires theory should be impaired by the fact that lithium is a very "open" metal. Measurements ${ }^{9}$ have revealed that considerable relaxation occurs in the lattice. As all electrostatic forces are very dependent on the distance between charges, a model not taking account for these effects can not be expected to explain the experimental results too rigorously.

The present results reveal a much greater diffusivity and a much smaller activation energy for $\mathrm{Au}$ in $\mathrm{Li}$ than has been found for $\mathrm{Ag}$ in $\mathrm{Li}$ (see Fig. 1). This seems remarkable, as both the atomic size and elasticity parameters are about the same for $\mathrm{Ag}$ and Au. Obviously any theory based solely on such parameters ${ }^{10}$ appears incomplete in this context.

The diffusion of gold has been measured (s. Table 1) both in lithium of normal composition and in lithium of reversed isotopic composition. As can be seen there is a distinct difference between the diffusion values in the two cases. The diffusion in the normal lithium is about $8 \%$ slower than in the reversed lithium. A different relation has been observed in self-diffusion ${ }^{4}$. These results naturally stimulates the interest for further study of isotopic differences in lithium.

This research has been supported by the Swedish Council for Applied Research. I wish to acknowledge stimulating discussions with Docent A. Lodding and to thank Professor A. KLEMM for his interest in this project.

9 R. A. Hultsch and R. G. Barnes, Phys. Rev. 125, 1832 [1962].

10 R. A. Swalin, Acta Met. 5, 443 [1957]; Z. Naturforsch. 23 a, 805 [1968]. 